\title{
A Prevalence Study of Acinetobacter Species and Their Sensitivity Pattern in a Tertiary Care Hospital Rajkot City of Gujarat (India): A Hospital Based Study.
}

\author{
Dr.Kunjal Vaja ${ }^{1}$, Dr.G.U.Kavathia ${ }^{2}$, Dr.Y.S.Goswami ${ }^{3}$, Dr.Shweta Chouhan ${ }^{4}$ \\ ${ }^{I}$ Microbiologist (Class-1), General Hospital Rajpipla, Gujarat, India \\ ${ }^{2}$ Associate Professor, Department Of Microbiology, P.D.U. Medical College, Rajkot, Gujarat, India. \\ ${ }^{3}$ Dean, Gmers, Medical College, Junagadh, Gujarat, India. \\ ${ }^{4} 3^{\text {rd }}$ Year Resident Doctor, Department Of Microbiology, P.D.U. Medical College, Rajkot, Gujarat, India.
}

\begin{abstract}
Background: Acinetobacter species has emerged as an important pathogen globally in various infections especially in hospital acquired infections.

Objectives: This study was conducted to determine the prevalence, and antibiotic resistance pattern of Acinetobacter species from various clinical samples.

Materials and Methods: The study included a total of 1000 clinical samples, collected from patients treated in P.D.U. Hospital Rajkot-a tertiary care hospital in Gujarat, India were included in study period from November 2012 to August 2014. Isolation, Identification and sensitivity of Acinetobacter species were performed by manual method.

Results: 48 (4.8\%) patients clinical samples showed growth of Acinetobacter species. Acinetobacter species isolation rate from blood were 24(50\%), pus 15(31.25\%), urine 4(8.33\%), CSF 2(4.18\%), sputum 1(2.08\%), plural fluid 1(2.08\%) and tracheal aspirate 1(2.08\%). Resistance observed to Meropenem was $41.67 \%$, Piperacillin -Tazobactum 58.34\%, Amikacin 52.09\%, Ceftazidime $79.71 \%$, Gentamicin $62.5 \%$ and Levoflaxacin $68.75 \%$. This data suggest that Acinetobacter isolated from hospital exhibits resistanas to multiple antimicrobial drugs.

Conclusion: The study will help to implement better infection control strategies and improve the knowledge of antibiotic resistance patterns of Acinetobacter species in our region.
\end{abstract}

Keywords: Acinetobacter species, antibiotics, multidrug resistance, nosocomial

\section{Introduction}

Members of the genus Acinetobacter are ubiquitous, free living organisms that prefer moist environment and can be easily obtained from soil, water, food and sewage [1]. They are usually considered to be opportunistic pathogens, and of recent have been reported to cause a number of outbreaks of nosocomial infections in hospitalized patients like septicaemia, pneumonia, wound sepsis, endocarditis, meningitis and urinary tract infection (UTI) [2,3]. Although acknowledged to be an opportunist in hospitalised patients, community acquired infections are reported and they can cause infections in virtually every organ system [4]. Interpreting the significance of isolates from clinical specimens is often difficult, because of the wide distribution of Acinetobacter in nature and its ability to colonise healthy or damaged tissue [5]. This study was undertaken to determine the prevalence, and antibiotic resistance pattern of Acinetobacter species from various clinical samples.

\section{Material And Method}

The study was undertaken in Department of Microbiology, P.D.U. Medical College, Rajkot (Gujarat, India), from period November 2012 to August 2014. Total 1000 clinical samples received in our laboratory from patients treated at P.D.U. Hospital Rajkot were included in this study. All the clinical samples were inoculated on MacConkey agar and blood agar. Inoculated plates were incubated at $37^{\circ} \mathrm{C}$ for $24-48$ hours. Colonies of Acinetobacter species were white/cream coloured, smooth, circular with entire edges on blood agar and nonfermenter with a pinkish tint on MacConkey agar. Microscopy showed gram negative coccobacilli on gram stain. Oxidase test was negative $[6,7]$. 
Identification scheme of Acinetobacter species.

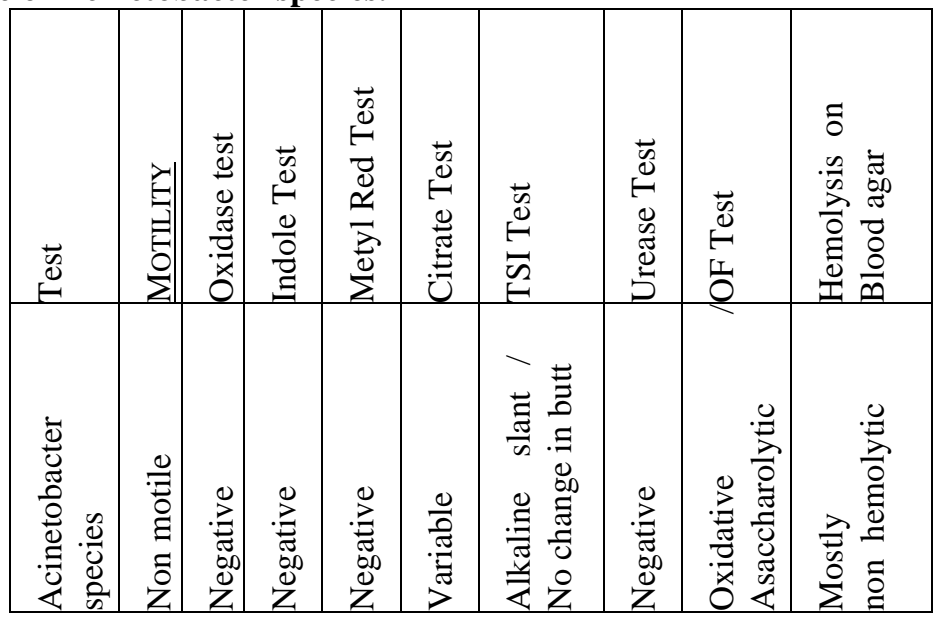

Antibiotic sensitivity testing of Acinetobacter species were performed by Kirby Bauer disc diffusion test. Antimicrobials tested were Amikacin, Gentamicin, Cefepime, Ceftazidime, Levofloxacin, AmpicillinSulbactam, Piperacillin-Tazobactam, Cotrimoxazole, Cefoperazon-Sulbactam, Tetracycline, Meropenem as per CLSI [8].

'Multidrug resistant (MDR) Acinetobacter spp.' is defined as isolate that is resistant to at least three classes of antimicrobial agents - all Penicillins and Cephalosporins (including inhibitor combinations), Fluoroquinolones and Aminoglycosides. 'Extensive drug resistant (XDR) Acinetobacter sp.' shall be the MDR isolate that is also resistant to Carbapenems. [9]

\section{Result}

Total 1000 patients clinical samples were included in present study, out of which 48(4.8\%) showed growth of Acinetobacter species.

Acinetobacter species were isolated highest from blood 24 (50\%), followed by pus $15(31.25 \%)$, urine $4(8.33 \%)$, CSF $2(4.18 \%)$, sputum 1 (2.08\%), plural fluid 1 (2.08\%) and tracheal aspirate 1 (2.08\%) (Table 1).

Table 1: Prevalence of Acinetobacter in various clinical samples.

\begin{tabular}{|l|l|}
\hline Clinical Sample & Isolation rate $(\mathbf{n = 4 8})$ \\
\hline Blood & $24(50 \%)$ \\
\hline Pus & $15(31.25 \%)$ \\
\hline CSF & $04(8.33 \%)$ \\
\hline Sputum & $02(4.18 \%)$ \\
\hline Plural fluid & $01(2.08 \%)$ \\
\hline Tracheal aspirate & $01(2.08 \%)$ \\
\hline
\end{tabular}

Number of Acinetobacter species were more from paediatric ward followed by surgical ward. Most of the isolates from paediatric ward was from preterm babies (Table 2).

Table 2: ward wise distribution of Acinetobacter Species .

\begin{tabular}{|l|l|}
\hline Ward & Isolation rate (n=48) \\
\hline Paediatric & $23(47.94 \%)$ \\
\hline Surgical & $13(27.08 \%)$ \\
\hline Medical & $05(10.41 \%)$ \\
\hline Obst and Gynec & $05(10.41 \%)$ \\
\hline TB and Chest & $02(4.16 \%)$ \\
\hline
\end{tabular}


Acinetobacter infection was found nearly equal in male 23(47.9\%) and female 25(52.1\%).

In Antibiotic Sensitivity Testing, highest resistance was observed to Cefepime (87.5\%) and lowest to Meropenem (41.67\%) (Table 3).

Table 3. Resistance pattern of Acinetobacter Species to different antibiotics.

\begin{tabular}{|l|l|}
\hline Drug & Resistance pattern \\
\hline Ampicillin Sulbactam(A/S)10/10ug/disc & $64.58 \%$ \\
\hline Ceftazidime(CAZ) 30ug/disc & $79.17 \%$ \\
\hline Levofloxacin(LE)5ug/disc & $68.75 \%$ \\
\hline Meropenem(MRP)10ug/disc & $41.67 \%$ \\
\hline Gentamicin(GEN)10ug/disc & $62.5 \%$ \\
\hline Amikacin(AK)30ug/disc & $52.09 \%$ \\
\hline Piperacillin Tazobactam(PIT)100/10 ug/disc & $58.34 \%$ \\
\hline Piperacillin(PI)100ug/disc & $64.59 \%$ \\
\hline Cefepime(CEP)30ug/disc & $87.5 \%$ \\
\hline Cefotaxime(CTX)30ug/disc & $75 \%$ \\
\hline Tetracycline(TE)30ug/disc & $66.67 \%$ \\
\hline Cotrimoxazol(COT)1.25/23.75ug/disc & $62.5 \%$ \\
\hline
\end{tabular}

\section{Discussion}

Acinetobacter spp. are the second most common Non-fermenting bacteria after Pseudomonas species that are isolated from human specimens, especially among nosocomial infections.[10] In recent years, this species has emerged as the causative agent of important nosocomial infections in the ICUs, which is probably related to the increasingly invasive procedures used, the greater quantity of broad-spectrum antimicrobials used, and prolonged duration of stay in the hospital. Development of resistance to antimicrobials is a major problem in the treatment of Acinetobacter infections.[11]

Isolation rate of Acinetobacter species in present study was $4.8 \%$, which is quite comparable with Lone et al (4.8\%) [12] and Mindolli PB et al(4.25\%) [13]. Higher prevalence rates of $14 \%$ and $9.6 \%$ among hospital isolates were observed by Mostofi et al. ( Iran) and Joshi et al. (India), respectively[14,15]. Acinetobacter spp. can colonize skin, wounds, respiratory and gastrointestinal tracts.[16] It is a pathogen of tropical and humid environment, but some species can survive environmental desiccation for weeks, a characteristic that promotes transmission through fomite contamination in hospitals.[17]

In present study Acinetobacter species were isolated highest from blood 24 (50\%), followed by pus 15 $(31.25 \%)$, urine $4(8.33 \%)$, CSF $2(4.18 \%)$, sputum $1(2.08 \%)$, plural fluid $1(2.08 \%)$ and tracheal aspirate 1 $(2.08 \%)$. Highest isolation was from blood, most of them were from preterm babies, where due to lower immunity, more chances of bacterial infection. In a study conducted by A. Asensio et al in 2008 Acinetobacter was isolated from respiratory tract $(42.2 \%)$, surgical wound $(15.1 \%)$, urinary tract $(12.9 \%)$, skin $(11.7 \%)[18]$.

In our study, $20.83 \%$ isolates were MDR \& $20.83 \%$ isolates were XDR. The other studies conducted by Bhattacharyya et al. in West Bengal [19] and Mostofi et al. in Tehran[14] reported the MDR isolates to be $29 \%$ and $54 \%$, respectively. Acinetobacter is ubiquitous in the hospital setting. Its ability to survive for long periods coupled with its ability to demonstrate a number of antimicrobial resistance genes has made Acinetobacter a successful hospital pathogen.[20,21]

Most of the patients who were admitted in our hospital had previously attended primary and secondary care hospitals and usually received combination of $\beta$-lactam antibiotics like third and fourth generation Cephalosporins along with Aminoglycosides or Fluoroquinolones. Majority of the isolates in our study were resistant to commonly used antibiotics such as Ceftazidime(79.17\%), Cefepime(87.5\%), Gentamicin(62.5\%), Amikacin(52.09\%), Levofloxacin(68.75\%), and Ampicillin/sulbactam(64.58\%). This suggest that MDR isolates are increasing, probably due to indiscriminate use of these antibiotics in healthcare settings. It is reemphasized that broad spectrum antibiotics should be used with caution. We found that, Meropenem(41.67\%) and Piperacillin/Tazobactam(58.34\%) were also showing resistance against this pathogen suggesting increased 
XDR isolates. Mostofi et al. in their study had reported resistant drug Meropenem (31\%) and Piperacillin/Tazobactam (40\%)[14]. Differences observed between the different studies, could be due to the methods, the resistance patterns and and the antimicrobial patterns used [22]. Although antibiotic resistance is a worldwide concern, it is first and foremost a local problem - selection for and amplification of resistant members of a species that are occurring in individual hospitals and communities, which can then spread worldwide[23] There are many measures that may impact on antimicrobial resistance; reducing and restricting the use of antimicrobials to only those situations where they are warranted, at proper dose and for the proper duration is the most appropriate solution.[24]

Carbapenems have been the drug of choice for treating Acinetobacter infections, but unfortunately, Carbapenem resistant Acinetobacter is becoming common worldwide[25, 26]

\section{Conclusion}

In the present study Acinetobacter spp. accounted for $4.8 \%$ of total culture. Resistance observed to Meropenem was $41.67 \%$, Piperacillin -Tazobactam 58.34\%, Amikacin 52.09\%, Ceftazidime 79.17\%, Gentamicin $62.5 \%$, Levoflaxacin $68.75 \%$ which suggested that Acinetobacter isolated from hospital exhibit resistance to multiple antimicrobial drugs.

Traditional typing methods like phenotyping and antibiogram typing have an advantage over genotyping as they are readily available in all clinical microbiology laboratories. Simple identification schemes and antimicrobial susceptibility testing provide a cost effective approach for typing Acinetobacter spp. Although above systems have certain limitations when compared to molecular methodologies, the distinction between resistant and susceptible Acinetobacters atleast, is useful for effective clinical management of the infection caused by this group of organisms.

Overall infections caused by Acinetobacter spp. Provide an impressive demonstration of the increasing importance of this genus as human pathogen because of the high potential of this genus to develop antibiotic resistance leading to a considerable selective advantage in environment with widespread and heavy use of antibiotic, especially with relation to hospital environment and nosocomial infections. To avoid resistance, antibiotics should be used judiciously and empirical antibiotic therapy should be determined based on local antibiotic sensitivity pattern of the prevalent organisms of the hospital.

\section{REFERENCES}

[1]. Gerner-Smidt P. Taxonomy and epidemiology of Acinetobacter infections. Rev Med Microbiol 1995;6:186-97.

[2]. Towner KJ. Clinical importance and antibiotic resistance o Acinetobacter spp. J Med Microbiol 1997;46:721-46.

[3]. Levi I, Rubinstein E. Acinetobacter infections-overview of clinical features. Acinetobacter : microbiology, epidemiology, infections, management In : Bergogne-Berezin I, Joly-Guilloo MI, Towner KJ, editors. Boca Raton, CRC Press. 1996;101-15.

[4]. Glew RH, Moellering RC, Kunz LJ. Infections with Acinetobacter calcoaeceticus (Herellea vaginicola) : Clinical and laboratory studies. Medicine 1997;56:79-97.

[5]. Henricksen SD. Moraxella, Acinetobacter and Mimae. Bacterial Rev 1973;37:522-61.

[6]. Colle JG, Fraser AG, Marmion BP, Simmons A. Practical Medical Microbiology. Churchill Livingstone 14th ed. 1996:294-6.

[7]. Koneman EW, Allen SD, Jande WM, Schreckenberger PC, Winn Jr WC. Colour atlas and text book diagnostic Microbiology. Lippincot $5^{\text {th }}$ ed $1997 ; 286-7$.

[8]. Clinical and Laboratory Standards Institute. Performance standards for antimicrobial susceptibility testing. $20^{\text {th }}$ informational supplement. M100- S20, Wayne; PA: USA. Clinical and Laboratory Standards Institute, 2010.

[9]. Dent LL, Marshall DR, Pratap S, Hulette RB. Multidrug resistant Acinetobacter baumannii: A descriptive study in a city hospital. BMC Infect Dis 2010;10:196.

[10]. Albrecht MC, Griffith ME, Murray CK, Chung KK, Horvath EE, Ward JA, et al. Impact of Acinetobacter infection on the mortality of burn patients. J Am Coll Surg 2006;203:546-50.

[11]. Bernards AT, Harinck HI, Dijkshoom L, van der Reijden TJ, van den Broek PJ. Persistant Acinetobacter baumannii? Look inside your medical equipment. Infect Control Hosp Epidemiol 2004;25:1002-4.

[12]. Rubina Lone, Azra Shah, Kadri SM, Shabana Lone, Shah Faisal. Nosocomial Multi-Drug-Resistant Acinetobacter Infections Clinical Findings, Risk Factors and Demographic Characteristics; Bangladesh J Med Microbiol 2009; 03 (01): $34-38$.

[13]. Preeti B. Mindolli, Manjunath P. Salmani, Vishwanath G and Hanumanthappa A.R; Identification And Speciation Of Acinetobacter And Their Antimicrobial Susceptibility Testing; AJMS (An US National Library of Medicine enlisted Journal), Al Ameen J Med Sci (2010) 3(4): 345-349 ISSN 0974 - 1143.

[14]. Mostofi S, Mirnejad R, Masjedian F. Multi-drug resistance in Acinetobacter baumannii strains isolated from clinical specimens from three hospitals in Tehran-Iran. Afr J Microbiol Res 2011;5:3579-82.

[15]. Joshi SG, Litake GM, Satpute MG, Telang NV, Ghole VS, Niphadkar KB. Clinical and demographic features of infection caused by Acinetobacter species. Indian J Med Sci 2006;60:351-60.

[16]. Getchell-White SI, Donowitz LG, Gröschel DH. The inanimate environment of an intensive care unit as a potential source of nosocomial bacteria: Evidence for long survival of Acinetobacter calcoaceticus. Infect Control Hosp Epidemiol 1989;10:402-7.

[17]. Rit K, Saha R. Multidrug-resistant Acinetobacter infection and their susceptibility patterns in a tertiary care hospital. Niger Med J 2012;53:126-8.

[18]. Asensio A, Canton R, Vague J, Calbo-Torrecillas F, Herruzo R, Arribas JL et al. Prevalence of infection by Carbapenem resistant A. baumanni in Spain (1999-2005). Enferm Infeec Microbiol Clin 2008 April;26(4):p.199-204. 
[19]. Bhattacharyya S, Bhattacharyya I, Rit K, Mukhopadhyay PK, Dey JB, Ganguly U, et al. Antibiogram of Acinetobacter spp. isolated from various clinical specimens in a tertiary care hospital, West Bengal, India. Biomed Res 2013;24:43-6.

[20]. Agoda A, Zarrelli R, Barllitta M, Anzaldi A, Di Popolo A, Mattaliano A. Alert surveillance of intensive care unit acquired Acinetobacter infection in a Sicillian hospital. Clin Microbiol Infect 2006;12:241-7.

[21]. Yu Yu, Yang Q, Xu Xw, Kong HS, Xu GY, G BY. Typing and characterization of carbapenems resistant Acinetobacter calcoaceticus - baumannii complex in a Chinese hospital. J Med Microbiol 2004;53:653-6.

[22]. Chakraborty B, Banerjee D, Chakraborty B. Acinetobacter baumannii: No more a choosy intruder? Indian J Med Sci 2011;65:344-8.

[23]. O'Brien TF. Emergence, spread, and environmental effect of anti-microbial resistance: How use of an antimicrobial anywhere can increase resistance to any microbial anywhere else. Clin Infect Dis 2002;34:S78-84.

[24]. MacDougall C, Polk RE. Antimicrobial Stewardship Programs in Health Care Systems. Clin Microbiol Rev 2005;18:638-56.

[25]. Towner KJ. Acinetobacter: An old friend, but a new enemy. J Hosp Infect 2009; 73:355-63.

[26]. Walsh TR, Toleman MA, Poirel L, Nordmann P. Metallo-beta-lactamases: The quiet before the storm? Clin Microbiol Rev 2005; 18:306-25. 